\title{
The Detrimental Effects of Surgical Smoke: Promoting Safety in an Operating Room Team-A Mixed Methods Approach
}

\section{Cobar SA, Stragg A and Abigail Mitchell RN*}

Simmons University, USA

*Corresponding author: Susan Aquino Cobar and Adam Stragg, Simmons University, USA, Tel: 716-930-0233; Email: susan.aquioncobar@simmons.edu

\section{Research Article}

Volume 3 Issue 3

Received Date: May 15, 2020

Published Date: June 18, 2020

DOI: $10.23880 /$ jqhe-16000164

\section{Abstract}

Background: Electrosurgical or electrocautery technology is one of the most widely used in the world, yet no federal agency has mandated the use of electrosurgical filter devices for the protection of surgical teams. Currently, there are 500,000 operating room workers in the U.S.

Objective: To identify and appraise perceived barriers and benefits that impact implementation of a hospital-approved electrosurgical filter device that reduces the exposure of surgical smoke by a surgical team. Utilize that data to implement a filter device and collect feedback.

Methods: The study is a quality improvement project that involved mixed methods. Because there were no patients involved, an institutional review board approval was deemed unnecessary. The operating room staff in an acute care hospital completed a nine-question survey to determine the pre-intervention perceptions of the staff. There were 53 pre-surveys handed out and 42 of them were returned-a $79 \%$ response rate. After the implementation of the device, focus groups were organized to collect feedback.

Findings: The main barrier expressed by the operating room staff was perceived cost of a system that would be effective in surgical smoke removal. The main perceived benefit from the use of an electrocautery filter device was the decrease in surgical smoke.

Conclusion: Four out of the 11 in the surgeon group expressed overall satisfaction with the filter device. Seven of the 20 (35\%) who provided feedback said they were satisfied and that same percentage expressed looking forward to future use of the filter device. Thirty percent (30\%) of respondents voiced reports of no smell, and $25 \%$ said they felt the filter device was useful in reducing surgical smoke.

Keywords: Smoke; Surgical procedure; Conventional Masks; Health Hazards

Abbreviations: NIOSH: National Institute for Occupational Safety and Health; SS: Surgical Smoke; OR: Operating Room; PAHs: Polycyclic Aromatic Hydrocarbons; HBM: Health Belief Model; OSHA: Occupational Safety and Health Administration; CDC: Centers for Disease Control; LEV: Local Exhaust Ventilation; ULPA: Ultra-Low Particulate Air; VOCs: Volatile Organic Compounds; ACS: American Cancer Society; PPM: Parts Per Million; LEEP: Loop Electrosurgical
Excision Procedures; UK: United Kingdom; JCAHO: Joint Commission on Accreditation of Healthcare Organizations; ANSI: American National Standards Institute; CRNAs: Certified Registered Nurse Anesthetists; WHO: World Health Organization; ICN: International Council of Nurses; APRN: Advance Practice Registered Nurse; ACCN: Association of Critical-Care Nurses. 


\section{Introduction}

Electrocautery, diathermy, or electrosurgical technology is widely used for cutting and coagulating tissues and vessels in operating rooms worldwide. This technology was developed by Harvey Cushing and William T Bovie. Saito, et al. [1] described its use as common due to the many advantages such as the reduction in surgical time and intraoperative bleeding, as well as improvement of visibility of the surgical field. As a result of temperatures of more than $100^{\circ} \mathrm{C}\left(212^{\circ} \mathrm{F}\right)$, products generated from use of an electrocautery device are water vapor and surgical smoke (SS). Authorities became acquainted with the dangers of SS when the National Institute for Occupational Safety and Health (NIOSH) published its health hazard evaluation report in 1985 in which authorities acknowledged that SS contained dead and living cellular material, blood fragments, bacteria, viruses, toxic gases and vapors [2]. Surgical smoke components, at certain levels similar and at others worse than cigarette smoke, have been shown to be detrimental, yet no federal agency has taken the step to declare the exposure of surgical smoke as dangerous to healthcare workers' health (Figure 1). To date, only Rhode Island and Colorado have passed a law addressing the dangers of surgical smoke exposure by requiring smoke evacuation systems in each operating room [3].

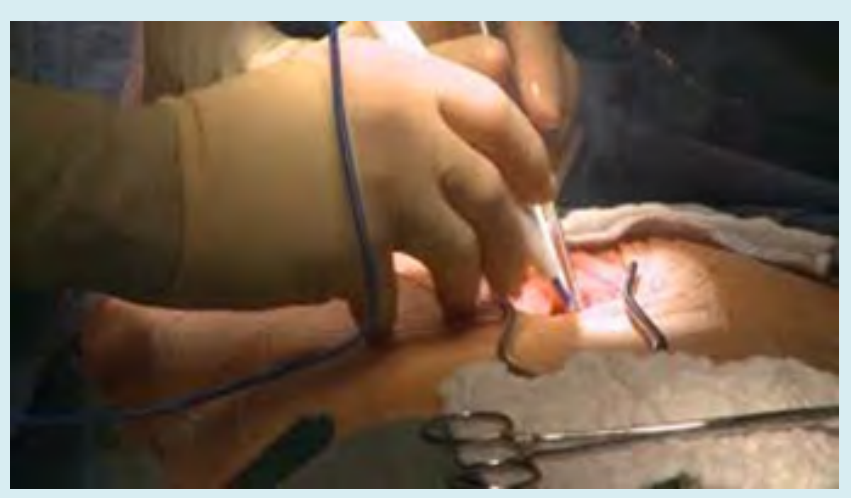

Figure 1: surgical procedure.

The emphasis of this literature review is to delve into the components of surgical smoke, to investigate the studied effects of those components on the overall health of operating room (OR) staff and patients, and to explore the different strategies utilized to decrease the exposure to surgical smoke. While many journal articles combined results of surgical smoke as a product of diathermy/electrocautery devices with that of laser and ultrasonic ablation, their effects are quite different. Laser ablation is newer, it yields larger particles and when compared to diathermy, the number of intact cells is lower [4]. Due to highly publicized cases of effects from SS resulting from laser ablation, there is higher compliance with personal protective equipment in conjunction with its use than with the use of electrocautery or ultrasonic ablation. Furthermore, and according to Saito, et al. [1], SS consists of polycyclic aromatic hydrocarbons (PAHs), hydrogen cyanide, formaldehyde, and carbon monoxide-many of which are carcinogenic compounds.

Although N95 masks are important components of personal protective equipment in lowering the exposure of staff to surgical smoke, they are second to the consistent use of efficient smoke evacuation systems [5]. The particle size range from electrosurgical technology use can be from less than 10 nanometers ( 0.01 micrometers) to 200 micrometers, which can easily diffuse through conventional masks that Katoch, et al. [6] assured only filter particulate matter larger than five (5) micrometers while high-filtration masks can filter out particles larger than 0.1 micrometers [7]. Conventional masks, York, et al. [5] commented, are best for bodily fluid splashes. Okoshi, et al. [2] conceded that any type of mask that is worn too long or too loose is ineffective for any protection. And although high-filtration masks can capture the smallest of bacterial particles, viral particles-such as HPV (human papillomavirus) from condylomas which are even smaller-may not be filtered out. In fact, high-filtration masks may also hinder breathing-lowering compliance rates. The AORN considers consistent, systematic use of smoke evacuation equipment paramount for keeping OR staff healthy and productive so they may tend to patients who rely on the team's expertise at a uniquely vulnerable moment.

\section{Research Question}

How can the implementation of the Health Belief Model increase the use of an electrosurgical filter device, a form of smoke evacuation system, to reduce the amount of surgical smoke affecting healthcare workers?

\section{Research Problem Statement}

In many surgical specialties, an electrosurgical device is an excellent method to help cauterize and coagulate tissue. As noted in Osman, et al. [8] electrosurgical units are implemented in multiple surgeries and help to coagulate, cut, and ablate tissue in order to prevent increased bleeding. However, this device generates surgical smoke that puts healthcare workers at risk of harmful health hazards. According to Bree, et al. [9] surgical smoke produces dangerous chemicals, viruses, and bacteria which can be adequately prevented with smoke evacuation devices. The goal of this project is to implement constructs of the Health Belief Model to increase the use of a hospital-approved electrosurgical filter device that reduces the exposure of surgical smoke by a surgical team. 


\section{Nursing Theorist-The Health Belief Model by the U.S. Public Health Service}

The main constructs of the Health Belief Model (HBM) are perceived seriousness, perceived susceptibility, perceived benefits, and perceived barriers. These constructs are modified by certain variables that influence the perception such as education, age, culture, socioeconomic status and motivation. Perception is awareness; however, actions are related to behaviors that are influenced by cues to action and self-efficacy [10]. The HBM framework will be implemented to increase the use of a filter device on an electrocautery filter tool. The goal of the project, which increases awareness of the effects of surgical smoke, is for OR staff to consistently use a filter device reducing their exposure to SS.

With the support of an operating room manager and educator, and utilizing the Health Belief Model, the team will educate the operating room team, including surgeons, physician assistants, anesthesiologists, nurse anesthetists, surgical technologists, scrub technicians and circulating nurses, of an acute care hospital in Maryland, based on a preliminary survey. The education will consist of evidence linking exposure of surgical smoke and effects on health. Once the education has been completed and a go-live date is set for consistent use of a filter at all procedures using an electrocautery device, focus group interviews will take place to explore resultant themes. The goal is to correlate an increase in awareness of dangers to surgical smoke exposure by this surgical team to increased compliance of filter device use and the associated levels of staff satisfaction, as well as a decrease in physical symptoms.

\section{Literature Review}

Healthcare-related literature was accessed focusing on manuscripts that explored negative outcomes in response to the exposure of operating room staff to surgical smoke as a result of the utilization of an electrocautery device with either inconsistent or without use of a smoke evacuation system. CINAHL, PubMed and Google Scholar were the databases utilized and the keywords for conducting the search included: surgical smoke, surgical plume, diathermy, diathermy plume, surgical smoke evacuation, surgical staff, barriers, compliance, safety measures, negative outcomes, negative effects, negative impacts, operating room, operating theatre, and electro surgery. Other sources incorporated into the literature review were the AORN, Occupational Safety and Health Administration (OSHA) [11], Centers for Disease Control and Prevention (CDC), NIOSH websites. The time period covered in the search was 2013 to January 2020, encompassing international literature, to ensure the most relevant, updated research was reviewed for the purpose of the project.

\section{Surgical Smoke Components}

Based on the 1980s report from the National Institute for Occupational Safety and Health and since then, many studies which have discussed surgical smoke, it is known that surgical smoke provokes symptoms in those who breathe it, whether knowingly or by accident. It is also known that cells and viruses present in plume are likely the cause of various symptoms and in the case of these, size matters. Lindsey, et al. [4] included in their systematic literature review a study in which particle sizes from laser, ultrasonic and diathermy tissue ablation were compared, and the original researchers concluded that diathermy ablation (by electrocautery device) generated the smallest particle sizes. In fact, in diathermy, the number of intact cells generated is higher than in laser surgery [4]. Lee, et al. [12] conducted various experiments using tissue from breast reduction surgeries and one belowknee amputation surgery to measure the number of particles, the average mass concentration of particles and the average count median aerodynamic diameter before, during and after use of an electrocautery device using 1) no local exhaust ventilation (LEV), 2) control with wall irrigation suction unit with in-line ultra-low particulate air (ULPA) filter, or 3) with a smoke evacuation system, and comparing findings to 4) the background (an empty OR).

Several instruments, including an airborne particlecondensation particle counter, a scanning mobility particle size, a light-scattering laser photometer, and an aerodynamic particle size and Biotrak were used during the procedure in the operating room. Additionally, research assistants donned evacuated canisters attached to a holster, fastened to a belt worn by each person. The average particle concentration which encompassed grab, area and personal sampling, without LEV was $74-12,200$ particles $/ \mathrm{cm}^{3}$. With LEV, the average particle concentration decreased to 300 3,900 particles $/ \mathrm{cm}^{3}$. When comparing the amounts and concentration of particles, Lee, et al. [12] concluded that the consistent use of any type of local exhaust ventilation would substantially reduce, although not eliminate, the number of airborne particles and volatile organic compounds (VOCs), entities that easily become gases or vapor.

Strengths of the Lee, et al. [12] study included that specific instrumentation, manufacturer and model numbers were specified. Some limitations were that the experiments were done during 15-minute electro cauterization followed by 30 minutes of particle analysis. Lee, et al. [12] noted that the level and composition of VOCs would likely vary by tissue types, surgical technique, type and energy level of surgical devices, as well as the sampled 45 minutes which is far lower than an actual exposure to SS by a healthcare worker.

Lee, et al. [12] further indicated that more than 150 
chemicals, as well as VOCs, found in surgical smoke included acetaldehyde (carcinogen), acrolein, acetonitrile, benzene (carcinogen), formaldehyde (carcinogen), hydrogen cyanide, polyaromatic hydrocarbons (carcinogen), styrene, toluene (carcinogen), xylene, in addition to 600 chemical compounds, which substantially increases the health stakes for OR staff. While the type of molecule is very important, the size of those molecules is crucial when relating this fact to health effects of OR personnel. Schultz, et al. [13] warned that the real danger lies in the inhalation of nanoparticles or "ultrafine" particles smaller than 0.1 micrometer (less than 100 nanometers), which make up $80 \%$ of SS. Schultz, et al. [13] explained that particles of that size are not effectively phagocytized by alveolar macrophages and have access to central and lymphatic circulation systems which travel to the rest of the organs. Over time, and without the use of adequate smoke evacuation systems, the body endures exposure to a level of chemicals similar or worse than smoking unfiltered cigarettes (Figure 2).

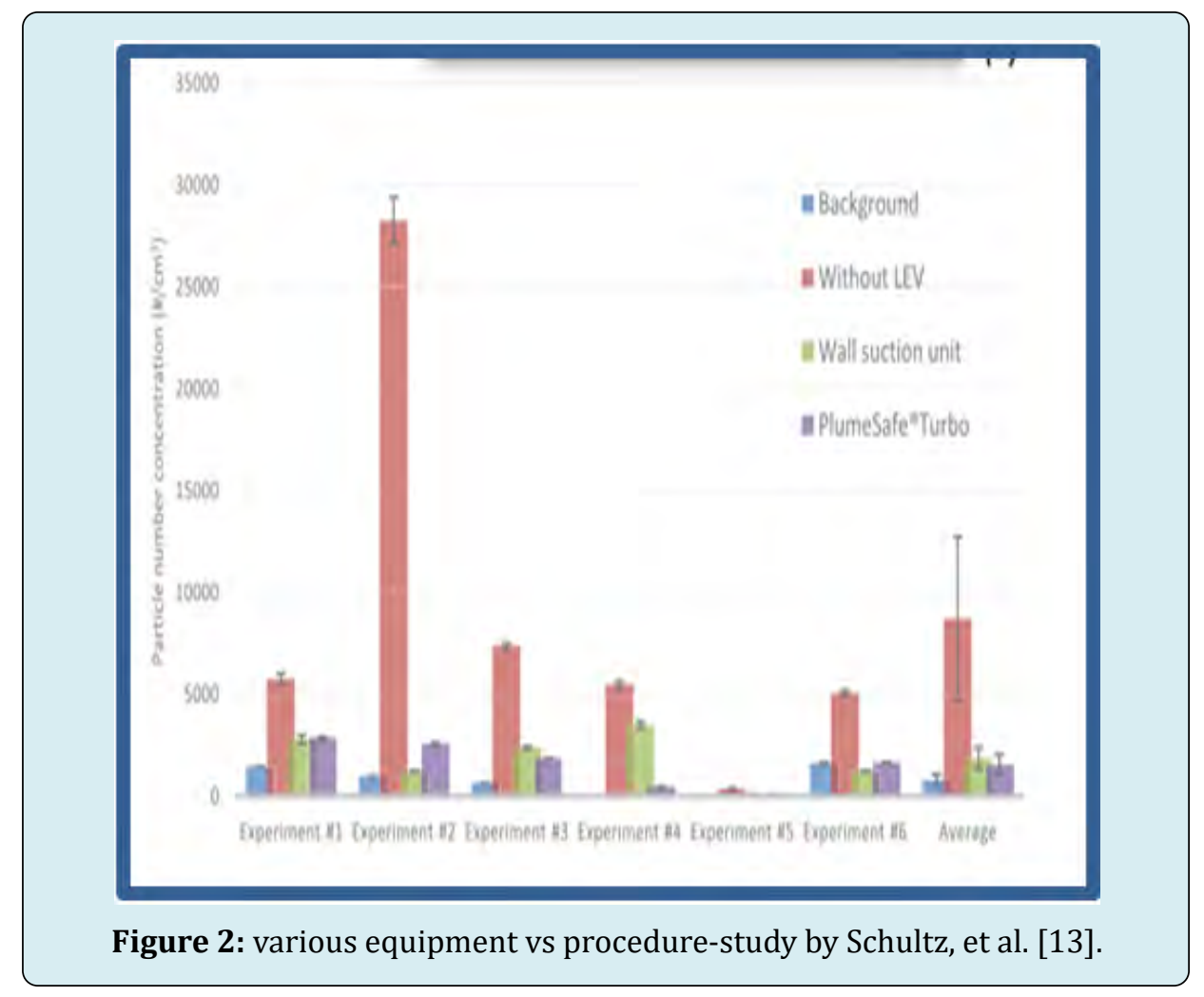

Mysore, et al. [6] specified that surgical smoke is composed of $95 \%$ water and $5 \%$ particulate matter. Mysore, et al. [6] assured that SS is a vector for infectious material including HPV, other malignant cells, and bacteria which have been studied in animals. Additionally, many compounds, of which many are known and probable human carcinogenic entities as demonstrated by the American Cancer Society [ACS] [14], are found in SS including xylene, styrene, ethyl benzene, toluene, carbon disulfide, in addition to compounds that displace oxygen from blood such as hydrogen cyanide and carbon monoxide, which at high levels are poisonous gases.

\section{The Operating Room-Staff Position}

The differences in time of exposure to surgical smoke as well as proximity to the source of it affect health outcomes irrespective of titles and roles. In a typical procedure, a chief surgeon, a surgeon assistant, a circulating nurse, an anesthesiologist, one or two surgical technicians and even a medical device representative may be present as well as others depending on the complexity of the surgical case. Saito, et al. [1] asserted in their cross-sectional study of 46 subjects, 10 of which were nurses, and 36 technologists and nurse assistants, all working in the surgical center of Londrina State University in the state of Paraná, Brazil, that nurses remained in the operating room intermittently. Surgical technologists and non-surgical technologists nursing professionals remained in the room from receipt of the patient in the OR to transfer of the patient to the postanesthesia care unit.

The surgeon who holds and operates the electrosurgical instrument is likely the closest healthcare professional to the source of surgical smoke. Okoshi, et al. [2] proposed that 
surgeons who worked 20-40 cm (approximately eight to 16 inches) from the incision and source of smoke experienced the highest concentration of SS. Despite their proximity to high doses of surgical smoke, overall their total exposure is decreased because they may operate occasionally throughout the week, while OR staff such as nurses, anesthesia providers and other personnel remain longer periods in the OR and experience a higher susceptibility to SS and its effects. This claim was supported by Tan, et al. [7] who stated that United States nurses and anesthesiologists/anesthetists, by nature of their role, spend more time in the operating room than surgeons, ancillary workers and orderlies. Notwithstanding, Lindsey, et al. [4] reported that in the United States, the OR members with the highest exposures to ultra-fine particles over short periods were surgeons and their assisting surgical staff.

Based on observations and the studies mentioned, it would be logical for surgeons to realize they are risking their health, just as much or more than the other staff. An observational study by Tseng, et al. [15] demonstrated that on average a surgeon was exposed to 16 carcinogenic PAHs and that group also had the higher risk of carcinogenic exposure due to their proximity to SS from electrocautery use when compared to anesthesia providers. The hazardous exposure was measured during 14 mastectomies, which required prolonged use of electrocautery technology, and particles in the SS were measured in two separate hospitals. Tseng, et al. [15] concluded that the more time spent in the $\mathrm{OR}$, the greater risk of vulnerability to harmful chemicals.

\section{Common Symptoms Related to Surgical Smoke Exposure}

As we learn about the types of molecules found in surgical smoke, the symptoms reported by operating room staff can be correlated to their exposure to those molecules at their place of work. Okoshi, et al. [2] specified the specific symptoms related to short- and long-term exposure to some of the compounds found in SS. For example, the effects of short-term (15 minutes) exposure to benzene in SS are eye, nose and respiratory tract irritation, headache, dizziness, and nausea. Exposure to low concentrations of benzene, long-term (eight hours), include anemia which will eventually become leukemia as a cumulative effect, and harm to reproductive organs [16]. High concentrations of benzene (exceeding OSHA limits) are noted in diathermy smoke during colorectal surgery, stated Okoshi, et al. [2].

Hydrogen cyanide, a colorless gas, in short-term exposure, causes headache, weakness, throat irritation, vomiting, dyspnea, lacrimation, colic, nervousness after absorption through skin and lungs, Okoshi, et al. [2] revealed. Toluene, which is well-absorbed via inhalation, irritates the eyes and the respiratory tract. In animal studies, toluene affects the central nervous system and in high levels, causes teratogens. The short-term exposure to acrylonitrile causes eye irritation, nausea, vomiting, headache, sneezing, weakness and light-headedness. Long-term exposure to acrylonitrile, as tested in animal studies, causes cancer and in humans, it is associated with higher incidence of cancer. Repeated and prolonged exposure of acrylonitrile on the skin produces irritation and dermatitis. The OSHA upper limit for this compound is two (2) parts per million (ppm) and the exposure levels of typical OR staff is $1.6 \pm 1.0 \mathrm{ppm}$ (OSHA, n.d.).

Researchers in the Saito, et al. [1] study collected consent forms from 46 subjects yielding a 100\% response rate. Each day after a shift, the respondents filled out their demographic information and responded to questions related to symptoms which have been associated with exposure to SS in past studies. The surgical and non-surgical technologist (OR nurses) reported higher rates of eye irritation, nasal mucosa and oral cavity irritation, and headache, all rates showing statistical significance $(\mathrm{p}<0.05)$. In animal studies, according to Katoch, et al [6], there was various objective data linking exposure of surgical smoke with a confirmed risk of direct physical injury, infection transmission and mutagenesis. A study referenced in Lindsey, et al. [4] in which 777 OR nurses were surveyed, $22.9 \%$ reported sinus infections/problems (vs. $10.3 \%$ of general population), $24.2 \%$ reported allergies (vs. $18.4 \%$ ), $10.9 \%$ asthma (vs. $6.4 \%$ ) and $9 \%$ bronchitis (vs. $4.5 \%)$.

An international study completed by Asdornwised, et al. [17] in Thailand used two surveys with a purposive sampling method which evaluated symptoms in 377 operating room nurses-nurses with two years' experience, trained as perioperative specialists or having attended an operating room nurse management conference. A total of 450 surveys were sent out and 377 were returned to researchers via mail or email (84\% response rate). One hundred percent $(100 \%)$ responded that in their place of work, there was little or no use of central smoke evacuation systems. Eighty-two percent $(82 \%)$ responded that there was little or no use of a portable smoke evacuation system. Fifty-six percent (56\%) reported little or no use of wall suction with in-line filters and $63.7 \%$ reported little or no use of laparoscope with evacuation/ filtration systems. Headache and sore throat were the most common symptoms reported, however, cough and/or sneezing severity was higher. Overall, the symptom intensity was low. A strength of the survey conducted by Asdornwised, et al. [17] was that in order to attain a confidence interval of $95 \%$, they needed 366 responses in the OR nurse population of 7,500. They also conducted a pilot study using 30 participants. The reliability of the survey tool, measured by Cronbach's alpha, was 0.80 . Also, the content validity score 
was one, after a chest medicine doctor, a thoracic surgeon, and a perioperative specialist nurse validated the tool. Weaknesses of the Asdornwised, et al. [17] study is that the types of surgery, specific roles in surgery, or time in surgery were not stratified, as these will affect the types of molecules and compounds and the proportion of each in SS, as well as the acuity of exposure.

Lindsey, et al. [4], in their systematic literature review, examined the types of tissues and the levels of chemicals most associated and they found that ablation of muscular tissue produced higher levels of aldehydes and ketones while ablation of liver and fatty acids produced high levels of carbon monoxide and hydrochloric acid. Lindsey, et al. [4] additionally, described that electro cauterization of epidermal tissue yielded increased amounts of xylene, toluene and ethyl benzene. Reduction mammoplasty produces high levels of toluene and furfural (levels above permissible exposure limits). Furfural is a solvent used in the petrochemical industry and is a respiratory irritant in the gaseous form. Human abdominal and transurethral surgery issue aerobic aerosol consisting of high levels of cyclohexane and cardiotoxic compound hydrogen cyanide and known carcinogens: 1,3 butadiene, vinyl acetylene and acrylonitrile. Interestingly, Lindsey, et al. [4] also acknowledged that ambient air (background) in the OR contains certain levels of toluene and formaldehyde (both known carcinogens) and those levels were not measured prior to the experiments.

Lindsey, et al. [4] adequately illustrated exposure by healthcare workers to high concentrations of toluene, again, a known carcinogen, in breast surgery. In general, levels produced measured $2.48-5.50 \mathrm{mg} / \mathrm{m}^{3}$, and levels are higher in radical mastectomies. A study referenced indicated the amount of toluene in single breast surgery is in excess of one $\mathrm{mg} / \mathrm{m}^{3}$. Cigarette smokers are exposed to about one $\mathrm{mg} /$ $\mathrm{m}^{3}$ daily. In general, people are exposed to about $0.3 \mathrm{mg} / \mathrm{m}^{3}$ toluene from the environment alone. The NIOSH permissible limit is less than $375 \mathrm{mg} / \mathrm{m}^{3}$ over a 40 -hour work (OSHA, unknown). The higher the temperatures and the shorter the procedure, the higher amounts of toxic chemical components was found, according to Lindsey, et al. [4] Limitations to the study were that small samples were utilized in most of the referenced studies and animal tissue was used instead of human tissue.

\section{Long-term Health Effects}

As people live longer, they also work more years in their profession. The current retirement age for those born after 1960 is 67. Noting this is significant because some operating room personnel may potentially work as long as 47 years if a surgical technologist completes training in 24 months after graduating high school. It is clear based on studies of exposure to cigarette smoke that the longer OR staff are exposed to SS, the effects can be devastating. Saito, et al. [1] expressed that long-term effects of exposure to SS included cancer, neurological, cardiac and respiratory disease and that damage extent was proportional to the length of exposure and the cumulative effects. Influencing the consequences of exposure involved surgical technique, procedure types, tissue pathology, and energy type supplied by available equipment, power levels and other variables. Katoch, et al. [6] claimed that the deposition of particles measuring less than two micrometers in the lungs can lead to chronic irritation, emphysema, interstitial pneumonia, and bronchitis. While the common symptoms are similar in many studies, Schultz, et al. [13] emphasized that even more importantly, continual exposure to surgical smoke is connected to an increasing risk of developing Parkinson's disease, Alzheimer's dementia, collagen and cardiac diseases as well as lung, breast and prostatic cancers.

York, et al. [5] discussed additional health effects from surgical smoke exposure such as anemia, anxiety, cardiovascular dysfunction, colic, dermatitis, hepatitis, human immunodeficiency virus, hypoxia, lacrimation, leukemia, nasopharyngeal lesions, nausea, vomiting and weakness. York, et al. [5] additionally, described being aware of those findings especially in the context of exposure by the surgeons and anesthesia providers in which it was found that particle concentrations, specifically of PAHs, increased 40-100 times the baseline measurements with surgeons being exposed 1.5 times the level of the anesthesiologist. PAHs comprise a type of chemical compound that exists in coal, crude oil and gasoline and is a product of the burning of coal, oil, gasoline, wood, garbage, or tobacco. If inhaled, PAHs have been associated with lung cancer; when touched by PAHs, skin cancer can be initiated; and, if ingested, stomach cancer may ensue. PAHs are best known for their association with cigarette smoking Figure 3.

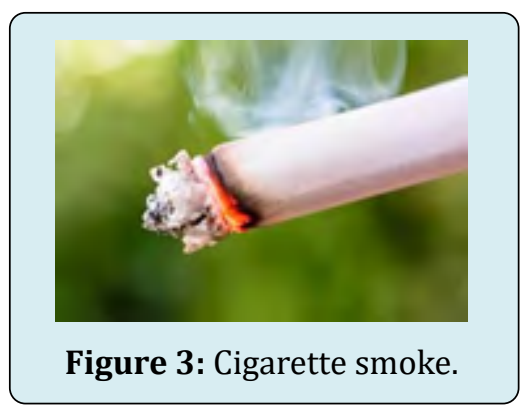

A case report about two gynecological surgeons authored by Rioux, et al. [18] noted that other than occupational exposure to the products of electro surgery and laser surgery in the operating room spanning 20 and 30 years for each person, they did not have any other strong risk factors for 
having developed squamous cell cancer. Patient 1, a 53-yearold gynecologist who had performed more than 3000 loop electrosurgical excision procedures (LEEP) on dysplastic cervical and vulvar lesions over 20 years complained of fatigue, a lesion on the right tonsil and a lump on the right neck. A biopsy staged the squamous cell cancer at T2N1M0, with HPV 16 as the source. Other than being HPV positive, once he experienced symptoms, no other rationale for developing squamous cell carcinoma could be found. He had been in a monogamous relationship with one wife, who tested negative for HPV. His only risk factor was being exposed to surgical smoke while performing procedures that involved known HPV lesions (Figure 4).

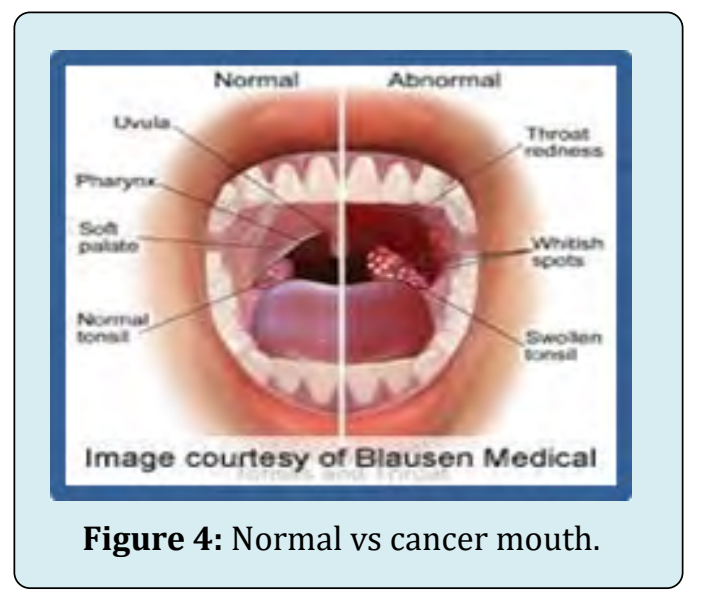

Patient 2 was a 62-year-old gynecology surgeon with 30 year experience with laser ablation and loop electrosurgical excision procedures. His symptoms included a sensation of having a foreign object in the throat for many weeks. The base of his tongue was biopsied and was found to show squamous cell carcinoma, also positive for HPV 16. His treatment consisted of excision of the lesion by laser with a modified bilateral neck dissection. His risk factors included occupational exposure, was married twice, and HPV status of his partners could not be established.

A systematic literature review undertaken by Bree, et al. [9] found in their 22 articles that though there is a clear association between components of surgical smoke and many reported symptoms, the articles failed to prove a causal relationship. Despite the lack of tangible evidence linking SS from electrosurgical devices to the morbidity and mortality of OR staff, Bree, et al. [9] determined that specific health problems may possibly be caused by SS components. Bree, et al. [9] contended that smoke exposure can be reduced with the use of LEV devices which may attach directly to electrocautery units or as a separate machine. They advocated for future studies to include the relationship of SS exposure to long-term health risks and effects on OR staff especially related to electrosurgical smoke. Bree, et al.
[9] also proposed to avoid electro surgery altogether when possible to increase protection of OR staff.

\section{Healthcare Worker Knowledge of Electrocautery Risks}

The acute care hospital in Maryland in which the project will take place and where each operating room is equipped with an electrocautery device filter connected to local exhaust ventilation, with a observed compliance rate of $0 \%$, it is suspected that a number of barriers exist to explain the poor compliance. Lack of knowledge, not enough experience with smoke evacuation systems, not having the training on the use of the filter, the OR hierarchy with the surgeon leading standards of practice among other reasons could be those barriers. In a cross-sectional study by Osman, et al. [8] 40 operating room staff of a public hospital in New Zealand was surveyed. Ninety-five percent $(95 \%)$ had three or more years of operating room experience. Seventy-five percent $(75 \%)$ had never attended any instructional session or class on surgical smoke evacuation methods, although $75 \%$ of them were aware of the different methods of surgical smoke evacuation. Ninety-seven-point-five percent $(97.5 \%)$ were unaware of any hospital surgical smoke control policy. Ninetyseven-point-five percent (97.5\%) were also concerned about the effects of surgical smoke exposure. One hundred percent $(100 \%)$ were also knowledgeable that a mask would not give any protection from surgical smoke exposure.

Barriers listed in Osman, et al. [8] for inconsistent use of surgical smoke evacuation methods were: Loud noise (25\%), cumbersome or not as manageable as other methods (75\%), perceived high cost (50\%), size or thickness of tool obscured user's view (50\%), and unsure of any limitations (5\%). Interestingly, a cryosurgeon who was interviewed by Osman, et al. [8] was reportedly surprised that others were not utilizing SS evacuation systems because he thought it was an expectation. He shared that it was part of his training in the United Kingdom (UK). Osman, et al. [8] concluded that the key to consistent use of smoke evacuation systems is education and policy. Limitations of the Osman, et al. [8] study is that it included a small number of participants. Similarly, in Lindsey, et al. [4] the frequency of smoke evacuation equipment use was influenced by 1) the surgeon's perception of hazard, 2) the surgeon's minimization for the need of evacuation procedures citing difficulty in managing larger equipment and decreased visibility of surgical field, and 3) not using it was routine for the staff. An AORN study referenced in the Lindsey, et al. [4] work, cited that $86 \%$ of surveyed perioperative nurses reported the main reason for non-compliance with smoke evacuation systems was the surgeon's resistance or refusal. Yet, as many studies have suggested, surgeons can be at highest risk for developing serious health issues. 
In a literature review by Holmes, etal. [19] which included studies in the United States and the United Kingdom (UK), barriers to compliance with smoke evacuation practices also involved surgeon refusal, staff attitudes, lack of managerial support and the complicated designs of smoke evacuation systems. Successful implementation of a SS evacuation program is linked to strong managerial leadership and adequate education. Strengths of the Holmes, et al. [19] review are that the generalizability is high because of the location of the studies (USA and UK) although weaknesses included that the surveys were cross-sectional with small sample sizes and low response rates. The surveys incorporated nurses' perceptions whereas surgeons' perspectives were not emphasized.

When policies are not clear, they are very difficult to enforce and so it is not surprising to observe staff independently interpret risk. When discussing personal protective equipment, with focus on masks, which are not the first line of protection against SS, rather smoke evacuation equipment is, one reason offered by staff in the Tan, et al. [7] study is that there was lack of confirmation that the patient had an infectious disease. Lindsey, et al. [4] pointed out that an issue with masks is poor fit. They also mentioned that the likelihood of using protective equipment is less in diathermy procedures than laser procedures even though diathermy procedures produce higher numbers of intact cells, probably bacterial, because, according to Lindsey, et al. [4] they found no studies that demonstrated transmission of viral particles in diathermy plumes. A reason for the difference in protection may be related to the use of laser having received more media attention in recent times.

While there may be some question as to whether there is a causal relationship between squamous cell cancer arising from exposure of human papillomavirus in surgical smoke, Rioux, et al. [18] alluded to studies of cattle having been inoculated by laser plume produced by the dissolution of HPV-positive tissue, inducing transmission of HPV and subsequent tumor growth. Okoshi, et al. [2] summarized their findings related to particle sizes: Smaller particles react chemically while larger ones react biologically. Nonetheless, frustration emerges as the public health movement to create smoke-free hospitals (in reference to cigarette smoking), refusing to translate that to the $\mathrm{OR}$, despite the abundance of studies reflecting that breathing SS is equivalent to smoking 27-30 cigarettes, and that ablation of one gram of human tissue (the size of a penny) is equivalent to the mutagenic effects of smoking six unfiltered cigarettes [7]. Even though now there is no doubt about the causal effects of smoking, some hospital administrators, surgeons, nurses and hospital workers continue to challenge the causal effects of SS exposure and persevere in resisting measures to protect $\mathrm{OR}$ personnel.

\section{Issues with Smoke Evacuation Systems}

Just as with surgical ablation technology that has its risks and benefits, as does a surgical procedure itself, there is justification in considering the drawbacks of utilizing a smoke evacuation system. Having knowledge of those weak points may assist in implementing a protocol to reduce exposure of SS. A pilot study completed by Kochera, et al. [20] examined the use of mobile smoke evacuation systems during the electro cauterization of porcine tissue, which is physiologically comparable to human tissue. Multiple settings were used in order to create different volumes of SS. The goal of the observational study was to monitor the efficiency of this type of smoke evacuation systems in protecting surgical staff from harmful SS.

Nine particles were found which were carcinogenic and toxic. Despite the use of the smoke evacuator, the levels of butadiene and benzene remained above the permissible levels set by NIOSH. Kochera, et al. [20] also tested the ability to filter out toxic chemicals by use of a standard mask and a 3M N95 mask (which has been discontinued as of 12/31/18). They concluded that both the modern mobile smoke evacuation systems as well as both masks were unable to reduce the number of inhaled VOCs. A limitation to the study is that a small number of samples were used, the pilot study took place in a laboratory and not a dedicated OR with a ventilation system, and the focus was on a few toxic particles although it has been established that SS contains several types of cells, many more chemicals, and blood fragments.

\section{Effects of Surgical Smoke Exposure on Patients}

Although the exposure of surgical smoke is mostly associated to operating room staff, patients are also exposed to its deleterious effects because 1) perforation of the skin provides an entrance for volatile organic compounds and 2) not all surgical procedures require general anesthesia which means that patients, too, can suffer from surgical smoke exposure. York, et al. [5] discovered that in laparoscopic surgery in which small incisions are made for the purpose of inserting surgical tools, SS is trapped within the abdominal cavity of the patient. The risks to the patient are various. Visibility of the surgical site is poor which is also disruptive to the surgeon; the patient's body is exposed to SS byproducts such as benzene. Carboxyhemoglobinemia, carbon monoxide that is bound to hemoglobin in blood, and port-site metastasis has been confirmed. The main recommendation from AORN to mitigate those risks, according to York, et al. [5] is to attach a SS filter to the port on the laparoscopic trocar with the widest lumen to effectively remove the smoke closer to the SS source. 
A study authored by Dobbie, et al. [16] was based on the implementation of a surgical smoke evacuation program in an 844-bed Magnet academic medical center with 44 operating rooms in New York City. Dobbie, et al. [16] decided on the project after studying the effects of SS exposure on OR staff and patients. In a study Dobbie, et al. [16] included in their manuscript, urine samples were collected pre- and postlaparoscopic cholecystectomies. The first urine was obtained five-12 hours post-surgery and contained increased levels of toluene and three-times the level of benzene. As the reader may recall, benzene can be leukemogenic and teratogenic. None of the patients had been exposed to cigarette smoke during the study.

In some procedures, such as Mohs surgery in which skin cancer is excised, patients are not intubated and remain awake. A study, also cited by Dobbie, et al. [16] surveyed patients after Mohs surgery in which 30 patients that were unable to see whether a smoke evacuator device was used. In fact, one was used with closure of the incision and was turned off during the actual surgery. All the patients reported awareness of an unpleasant odor during the Mohs procedure and $40 \%$ of patients reported that same awareness during the closure stage. Sixty-three percent (63\%) reported that use of the smoke evacuation device contributed to a more pleasant surgical experience. Patient satisfaction is a measure used by Centers of Medicare \& Medicaid Services.

\section{Increasing Compliance with Use of Smoke Evacuation Equipment}

All the studies already cited contained a variety of surgeries on different types of tissues by different surgeons presumably using different models of electrosurgical devices. In a quantitative study, utilizing consecutive sampling methods, Liu, et al. [21] compared the number of particles using one condensation particle counter in spine surgery, procedures which generally produce similar volumes and compositions of SS because they involve comparable techniques. The particle counter, controlled by the circulating nurse, who was positioned near the anesthetist and within five feet of the incision, was able to capture particles between 0.02 and one (1) micrometers. Using a power analysis, Liu, et al. [21] determined 25 surgeries with each type of smoke evacuator would be necessary to obtain statistical significance. After achieving institutional review board approval, the same senior orthopedic surgeon, with an assistant surgeon, operated on 51 consecutive patients in one OR with laminar airflow using a conventional electrocautery tool in the coagulate mode at an output power of 35 watts, with a method of smoke evacuation. In the first 25 spine surgeries, the para-incisional smoke evacuator, a broad, flat suction pad placed with the surgical sheets, was positioned within five centimeters of the incision. For the following 26 spine surgeries, the surgeon utilized a smoke evacuation pencil in which the evacuation canula is part of the tool and smoke is captured and removed as the surgeon used it. Both evacuators were set at medium for all surgeries.

Liu, et al. [21] found that after analyzing the data and comparing the level of SS particles using the Wilcoxon signed-rank tests and inter-quartiles, that both types of evacuators significantly reduced the number of particles in surgical smoke. The average volume of smoke (number of particles per $\mathrm{cm}^{3}$ ) was decreased by $59.7 \%$ and the peak smoke level decreased by $95.9 \%$ utilizing the para-incisional smoke evacuator. The average volume of smoke was lowered by $44.1 \%$ and the peak smoke level by $75.3 \%$ utilizing the smoke evacuation pencil.

According to York, et al. [5] the Occupational Safety and Health Administration [22] does not specify any standards related to the protection of healthcare workers from the effects of surgical smoke. Healthcare workers in ORs across the country number about half million $(500,000)$. The CDC, OSHA, NIOSH, and the Joint Commission on Accreditation of Healthcare Organizations (JCAHO) only provide recommendations, suggestions and guidance related to SS exposure, while AORN and other international bodies that represent the interests of the OR workforce, have demonstrated that ventilation in ORs alone is insufficient for the removal of harmful substances that may or have impacted the health of OR employees around the world. York, et al. [5] continued by stating that the American National Standards Institute (ANSI) recommends a minimum of 20 total air exchanges per hour with a minimum of four of those containing outside air which must be documented. Most ORs, York, et al. [5] pointed out, are equipped with wall suction systems which are only effective in the removal of fluids, not smoke and are sometimes far from the source of SS. In general, wall suction generates two (2) $\mathrm{ft}^{3} / \mathrm{min}$ of airflow while a typical smoke evacuator moves air $35-50 \mathrm{ft}^{3} / \mathrm{min}$. Wall suction only works efficiently (not at removing smoke) when the tips do not get clogged by fluid and tissue.

As with any safety protocol, costs are a consideration. York, et al. [5] referenced that the AORN has invested many resources in studying the effects of SS on OR staff, along with many other organizations. Their recommendation is for each OR to be equipped with a smoke evacuator outfitted with a 0.1 micrometer filter (an ULPA filter). Their other guidelines involve giving access to OR staff to educational resources about SS and its dangers. The AORN's goal is for OR teams to recognize that SS can be harmful to anyone in the OR, that OR teams should expect health organizations to provide a SS-free work environment, to have equipment available for the removal of SS, and for OR teams to collaborate with administration on developing policies that outline 
procedures for smoke evacuation.

It has been established that surgical smoke is detrimental. However, it is also a fact that there is a hierarchy in the OR in which surgeons are at the top, at most institutions. York, et al. [5] stated that the goal of transforming ORs to SS-free environments cannot be attained without surgeon buy-in. The data must be presented to that group, identifying which members of that team will support the changes and who will challenge them is as equally important as educating the staff. Okoshi, et al. [2] were aware of the importance of promoting surgeons' acceptance and collaboration in smoke evacuation practices. A study Okoshi, et al. [2] remarked on was a survey of Mexican surgeons. Fifty-eight percent of them developed lumps in the throat and $22 \%$ complained of a sore throat, all as a result of electrocautery smoke. These as well as the case series by Rioux, et al. [18] should solidify the perceived threat of SS exposure by surgeons.

The Association of peri Operative Registered Nurses guideline summary [23] also emphasized that the decision on whether the perioperative team should evacuate all surgical smoke should not rest on one individual practitioner. A gap analysis must be completed to ensure the needs of operating rooms. The guideline also stated that a multidisciplinary team should collaborate in order to select and order adequate SS safety equipment. In addition to surgeons, perioperative registered nurses and scrub personnel, an infection preventionist, biomedical/heating/ventilation/air conditioning systems engineers and materials manager all are essential for an organization to become SS-free.

Tan, et al. [7] recommended that staff education on the hazards of surgical smoke exposure, infection prevention practices and methods to minimize or eliminate surgical plume would increase compliance with use of smoke evacuation systems. Tan, et al. [7] continued that with support from management, frequent in-services provided by vendors of smoke evacuation equipment, regular audits, informative posters in the OR, staff room and hallways, along with simple, easy to follow policies are keys to ensuring compliance with smoke evacuation procedures.

\section{Methodology}

\section{Design}

The study is a quality improvement project that involved mixed methods. Because there were no patients involved, an institutional review board approval was deemed unnecessary. Authorization was obtained by the operating room director and manager of the OR in an acute care health system, which according to Hirshon, et al. [24] includes "health system components...to treat sudden, often unexpected, urgent or emergent episodes of injury and illness...Acute care encompasses a range of clinical health-care functions, including...surgery..." The team sought to assess perceptions of OR personnel regarding the dangers of surgical smoke, to educate them using the evidence, to use a team approach to change practice in order to increase the compliance of filter device use, and finally to gather team feedback once the intervention was implemented Figures $5 \& 6$.
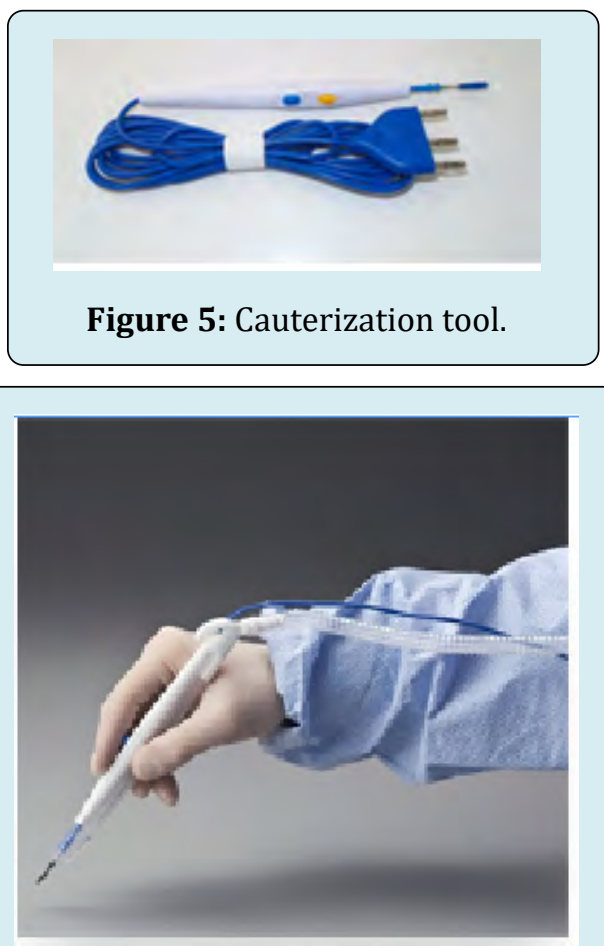

Figure 6: Cauterization tool with the surgical smoke filter device.

\section{Setting and Sampling}

The quality improvement project involved the operating room staff in an OR-in an acute care hospital. Convenience sampling was used to collect data over two weeks. Survey subjects included surgeons, anesthesiologists, nurse anesthetists, physician assistants, surgical assistants, scrub technicians (one was dually certified as surgical assistant and scrub technician) and circulating nurses.

\section{Data Methods}

A nine-question survey was drafted to determine the pre-intervention perceptions of the staff. The goal of this pre-survey was to assess the perceptions and knowledge of the operating room staff on the detrimental health effects of surgical smoke and the benefits of increasing the use of a smoke evacuation filter device. The pre-survey was distributed to the team along with a consent letter describing the purpose of the research project. There was a two-week 
period for staff to complete the survey. The survey contained two Likert-style questions, five single-answer questions and two open-ended questions. Questions were developed by the two student researchers utilizing the Health Belief Model as the framework and were based on the literature review. Return of the survey was deemed as consent for its use in data analysis. The intervention included an educational session for the nurses, scrub technicians, and surgical assistants about the harmful effects of surgical smoke. The education meeting also included a representative from the smoke evacuation filter device company who performed an in-service for staff about how to use the filter device. After the staff education and training, the filter device implementation occurred for two weeks and feedback was received through focus groups utilizing informal open-ended questions.

\section{Data Collection}

The surgical areas that were offered the pre-survey included general, gynecology, plastics, orthopedics, neurology, and cardiac surgery. There were 53 pre-surveys handed out and 42 of them were returned-a $79 \%$ response rate. The breakdown in responses were surgeons: 10 , surgical residents: 1 , anesthesiologists: 7 , Certified Registered Nurse Anesthetists (CRNAs): 3, circulating nurses: 9, surgical technicians: 5, physician/surgical assistants: 7. After the two weeks, the intervention took place.

The project was developed into a mixed methods tradition with post-intervention focus groups that were organized to collect data. Focus groups are "an active interaction among participants to explore views and opinions" [25]. There were 20 staff members that participated in using the smoke evacuation filter device and each gave back their verbal post-survey feedback. The breakdown in verbal post-survey responses was surgeons: 7, anesthesiologists: 1, CRNA's: 3, nurses: 4, surgical technicians: 3, physician assistants: 2 (Figure 7).

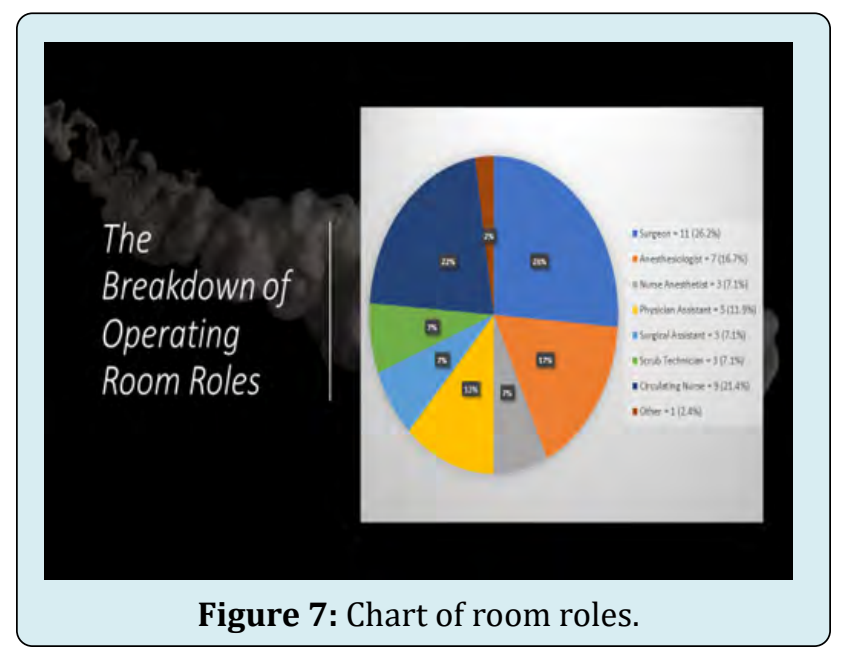

\section{Data Analysis}

Operating room teams are made up of various roles and are similar across the nation. Upon reviewing results, each of those roles was appropriately represented. Twenty-six percent $(26 \%)$ of respondents were surgeons while eleven $(21.4 \%)$ of them were circulating nurses. The next largest responding role was anesthesiologist at seven $(16.7 \%)$ and physician assistants representing $11.9 \%$ of total respondents (or five). The three smaller respondent roles were three each (7.1\%) of surgical assistants, scrub technicians, and nurse anesthetists (Figure 7).

The length of experience in their current role was extensive with $42.9 \%$ reporting 20 -plus years on the job. Twenty-three-point-eight percent (23.8\%) declared an 11-to15-year experience while there was an even split $(11.9 \%$ each) between those with 1-5 years and 6-10 years. The smallest portion (9.5\%) reported a 16-to-20-year experience while none reported less than one-year experience in their current role.

The perceived smoke impact prior to receiving any education as part of this project's interventions, $47.6 \%$ (20) reported a minimal impact of surgical smoke to their individual health, while $28.6 \%$ (12) communicated a moderate impact. The balance was evenly split between no effect and high effect at $11.9 \%$, or five each. The corresponding roles and length of experience will be discussed below.

As for the perception of what the best equipment or process that most effectively decreases the volume of surgical smoke, the clear majority (69\% or 29 ) indicated that an electrocautery filter device would fulfill that job. The next most voted method was the use of high-filtration surgical masks (16.7\% or 7$)$, room ventilation (11.9\% or 5$)$, followed 
by suction $(7.1 \%$ or 3 ) (an option that was written in by the three respondents), rounded out by regular masks $(2.4 \%$ or 1). None of the respondents perceived that N95 masks were useful in decreasing the amount of surgical smoke.

The team wanted to assess the level of satisfaction around pre-intervention practices of surgical smoke evacuation, and a question was designed specifically to address this point. Although not a majority, the highest reported opinions were somewhat dissatisfied (38.1\% or 16$)$ and neutral $(31.0 \%$ or 13) with $16.7 \%$ (7) indicating extreme dissatisfaction. About $4.8 \%$, or two, reported some to extreme satisfaction with pre-intervention methods. The team also wanted to correlate the satisfaction with pre-intervention electrocautery filter use which, in fact, showed that $54.8 \%$ (23), a clear majority, reported $0 \%$ use of said filter while the rest of the responses were split between an electrocautery filter use of 1-25\%, 26$50 \%, 51-75 \%$ and $76-100 \%$.

Supporting the findings of the Health Belief Model which declares that to effectively implement a change, an assessment of the perceived barriers and benefits can provide that knowledge is the reason behind the design of two open-ended questions that sought to explore those themes. As found in the literature review the larger themes relating the use of electrocautery filter devices and the perceived barriers were cost $(20.0 \%)$, lack of availability $(18.2 \%)$, surgeon preference $(12.7 \%)$ and no barriers (14.5\%). The perceived benefits also reflected the main purposes for the use of an electrocautery filter device which were less smoke (37.3\%) and reduction of impact on health $(27.5 \%)$. Other themes included complete removal of smoke $(5.9 \%)$, decreased smell $(5.9 \%)$ removal of smoke at the source (5.9\%), job/staff satisfaction (3.9\%), unknown benefits (3.9\%), improved visualization (3.9\%) and lastly, possible effectiveness, no benefits and multiple benefits (none specified), with $2 \%$ each.

\section{Staff Roles and Experience}

The single largest role surveyed was the surgeons. At $26.2 \%$ of the total operating room staff surveyed, with most of that subgroup (54.5\%) having over 20 years' experience in the operating room, surgeons' feedback was significantly reflected in the study. The next large group were the circulating registered nurses (21.4\%), comprised by over three-quarters $(77.8 \%)$ with eleven-plus years in the field. They were closely followed by anesthesiologists (16.7\%) with over $71.4 \%$ of that subgroup reporting sixteen-plus years' OR experience. One of the subjects surveyed reported certification in both surgical assisting and scrub technology.

\section{Staff Roles and Barriers}

The main barrier expressed by the staff by role was perceived cost of a system that would be effective in surgical smoke removal. In fact, four of the 11 surgeons (36\%) said their main concern was cost and three out of the seven anesthesiologists (about 43\%) said the same. Another four of the 11 surgeons (again 36.4\%) reported no barriers, as did two physician assistants $(40.0 \%)$ and one circulating nurse $(11.1 \%)$. Four of the nine circulating nurses $(44.4 \%)$, reported that the barrier was surgeon preference. Other barriers reported in the open-ended question included perceived noise, lack of training, comfort level, unknown, bulkiness, time for setup, complexity of surgery, none, distraction, management priorities, lack of education, lack of experience using one, doubted effectiveness and it is currently not mandatory.

\section{Staff Roles and Benefits}

The main perceived benefit from the use of an electrocautery filter device was the decrease in surgical smoke which was echoed by almost all roles (except for surgical assistants). Four out of nine surgeons (44.4\%) opined that there would be a reduction in surgical smoke. Exactly $66.7 \%$ of the circulating nurses and the surgical assistants perceived that one of the perceived benefits in the use of an electrocautery filter device was a reduction of impact on health. All three $(100 \%)$ of the scrub technicians agreed that a benefit would be a reduction in surgical smoke. Other benefits offered in the open-ended question were the absolute removal of smoke, improved visualization; unknown, removal of smoke at the source, possible effectiveness, job/ staff satisfaction, a decrease in smell, no benefits and one respondent wrote "multiple benefits" although none were specified (Chart 1).

\section{Staff Experience and Views on Surgical Smoke Impact}

One of the themes in the literature reviewed was the lack of knowledge of operating room staff regarding the impact of surgical smoke on individual and group health. Despite the group's substantial experience and time in the operating room, it was reported by $47.6 \%$ that the impact of surgical smoke was minimal, while $11.9 \%$ report no impact from it. About $28.6 \%$ said that there was moderate impact, while another $11.9 \%$ reflected a high impact of smoke on their health.

When further examining the subgroups, as expected the largest percentage of any of the groups were the anesthesiologists of whom $71.4 \%$ reported minimal impact, followed by surgeons of whom $54.5 \%$ who also declared minimal impact. Four out of the nine circulating nurses (44.4\%), also declared minimal impact from surgical smoke, while all the CRNAs reported either minimal (33.3\%) or 
moderate $(66.7 \%)$ impact. You may recall that both surgeon and anesthesiologist groups are the closest to the source of surgical smoke in the literature reviewed. The other groups did not stand out as having a prevailing view on the impact of smoke.

\section{Staff Experience, Role and Symptoms}

Having access to a highly experienced group which included a well-rounded representation of all the disciplines in a surgical team, it was important to collect their feedback on symptoms. The study found that about $41.5 \%$ of respondents felt that it was likely that any health symptoms can be attributed to exposure to surgical smoke. This was contrasted by the $26.8 \%$ who reported there was no relation of any health concerns or symptoms to surgical smoke. Another $22 \%$ said it was very unlikely that any symptoms were a result of said exposure. The smallest group of $9.8 \%$ reported it was very likely that these two concepts of symptoms and surgical smoke exposure were connected.

The higher percentages of any of the roles who reported any connection of physical symptoms to exposure to surgical smoke belonged to the surgeons. About $72.7 \%$ of the surgeons reported that symptoms were either very unlikely or likely to be related to surgical smoke. The group expressing a high likelihood of the connectedness between symptoms and surgical smoke were the circulating nurses, of which one-third shared that perception. Another third in that same group shared that there was no relation between the two. The entirety of the nurse anesthetist as well as the surgical assistant groups, reported the likelihood of that relationship. There was also one surgeon (representing 9.1\% of that group) who said that symptoms were very likely to be associated with SS.

\section{Staff Satisfaction, Roles and Filter Usage}

Upon further review of responses, it was found that over $54 \%$ of the respondents were either extremely or somewhat dissatisfied with pre-intervention methods of surgical smoke evacuation. When the satisfaction was compared to the preintervention usage of a filter device, it was found that out of the four respondents who responded to a high usage (76-100\% in the past week) of an electrocautery device, $75 \%$ of them reported some dissatisfaction and only one respondent said $\mathrm{s} /$ he was somewhat satisfied. On the other extreme, of those who reported $0 \%$ use of an electrocautery filter device, about $87.0 \%$ were either extremely dissatisfied, somewhat dissatisfied or neutral, the rest reported some or extreme satisfaction with pre-intervention methods of smoke removal. A similar spread of perceptions was noted when examining the responses between pre-intervention satisfaction and perceptions of smoke impact on individual health.

Probing the numbers for satisfaction with the preintervention system of surgical smoke removal in relation to roles, it was noted that five of the 11 surgeons (45.5\%) replied neutral, whereas six of the seven anesthesiologists (85.7\%) expressed either neutral or some dissatisfaction. When considering the circulating nurses, they all were either neutral, extremely dissatisfied (33.3\%), or somewhat dissatisfied (44.4\%). None of them reported some or extreme satisfaction. In addition, all three surgical assistants express some dissatisfaction. Only one surgeon and one physician assistant recorded extreme satisfaction.

\section{Post-Intervention Focus Group Themes}

Although the feedback by role was varied, none of the roles had more to report than the surgeon group. Their responses connected to almost all the themes (mentioned below) at least once. Four out of the 11 of that group expressed satisfaction with the filter device Charts $1 \& 2$. Seven of the total 20 (35\%) who provided feedback said they were satisfied and that same percentage expressed looking forward to future use of the filter device. Thirty percent (30\%) of respondents voiced reports of no smell, and $25 \%$ said they felt the filter device was useful in reducing surgical smoke in the operating room. Post-intervention themes which arose from the focus groups relating to the supported use of the available filter device included satisfaction, smoke reduction, absence of smell, interest in other filters, too thick or cumbersome, obstructive, convenient, quiet, increased safety, decrease in nausea symptoms, noisy, no barriers, past experience using, no issues operating and looking forward to continued use (Chart 2).

\section{Some quotes from the focus groups were:}

- Surgeon: "I really enjoyed using this filter...I would like to continue using this or a similar device moving forward."

- Nurse: "I personally get nauseous around surgical smoke, so I felt better when I did not smell the smoke."

- Scrub technician: "The filter device is a great thing...I would like to try the newer filter model that is slimmer and may be longer..."

- Physician assistant: "I fully support this initiative and would like to use this filter device more often."

- Surgeon: "I am in support to remove surgical smoke...I would like to trial the new and slimmer versions which are...easier to use."

- CRNA: “I really liked that I didn't smell anything, and it is a great safety precaution." 

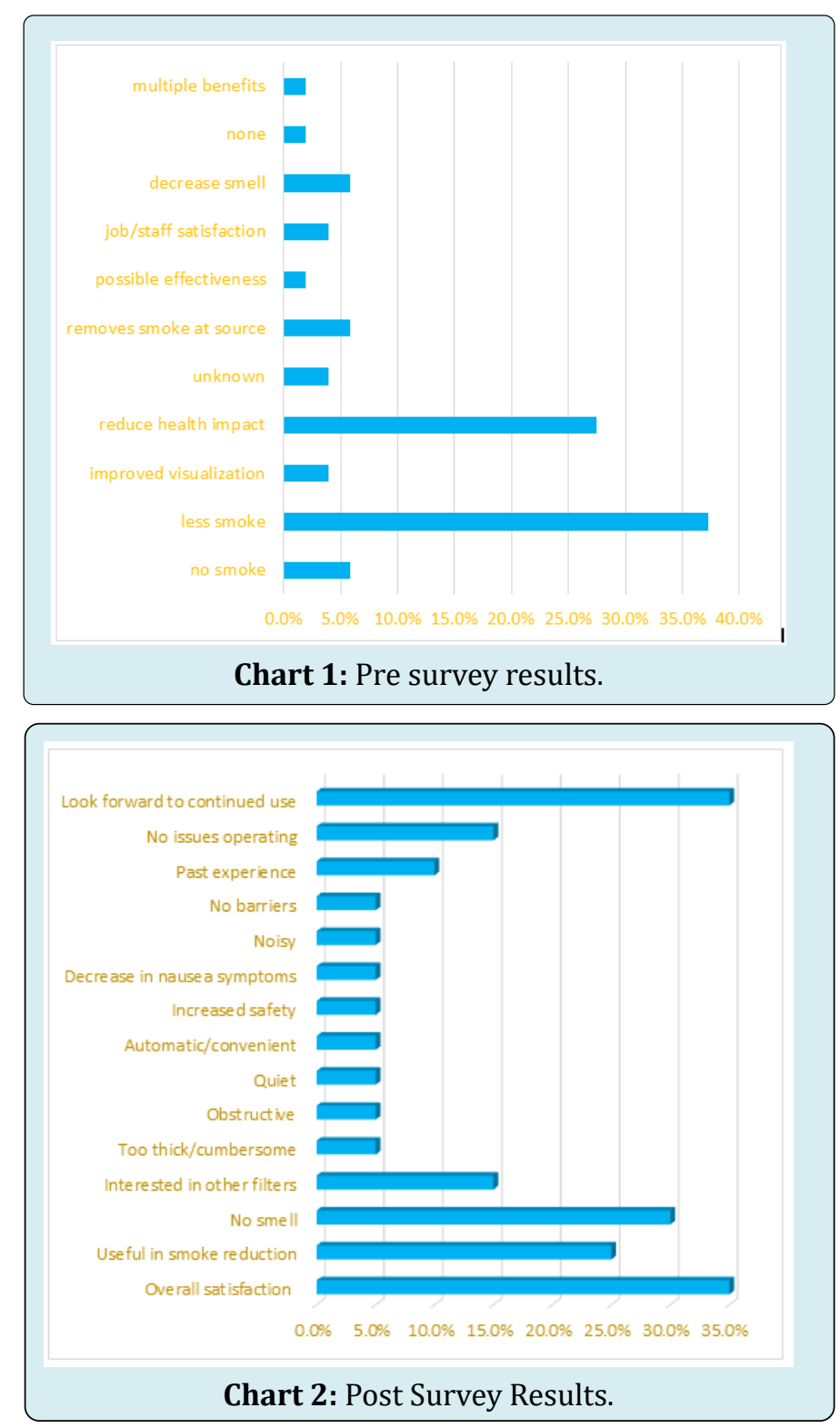

\section{Discussion}

Despite the varying perceptions regarding the impact of surgical smoke on health and symptoms, a majority (64.4\%) agreed that the most effective method of reducing surgical smoke was the use of an electrocautery filter device. Eight out of $11(72.7 \%)$ surgeons representing about three-quarters of them agreed on this. At a very distant second, high-filtration surgical masks received about $15.6 \%$ of the vote. One sole circulating nurse believed that the use of a regular mask was good enough to reduce exposure to surgical smoke. This makes the project team surmise that although the surgeons are aware of available smoke evacuation technology, no one else in the operating room has received significant education regarding the dangers of surgical smoke, although the team as a whole are knowledgeable that the most effective tool against that exposure are filters used in conjunction with electrocautery equipment. Another point to emphasize is that, like the findings in the literature review, the more common barrier for the use of an electrocautery filter device shared by four of the nine circulating nurses (44.4\%) was surgeon preference, while the surgeon group's main preoccupation was cost, system availability, and another four of the nine $(36.4 \%)$ reported that there are no barriers to the use of an electrosurgical filter device.

When considering the satisfaction of the team, specifically those who work closer to the source of surgical smoke, i.e. the surgeons, anesthesiologists, CRNAs and surgical assistants, the overall satisfaction is more negative than positive. This provided a good overall feel of the need for an intervention which reflected well on the hypothesis 
that this surgical team would benefit from education to increase use of a filter device. Mc Nicholas, et al. [26] demonstrated in their performance improvement project that healthcare team satisfaction is directly related to patient satisfaction. Nurse and patient satisfaction numbers were compared before and after a set of interventions that made modifications in which teams felt respected and heard. That data was then associated to the higher retention rates, better patient experience and cost-effectiveness.

\section{FNP Implications}

The World Health Organization (WHO) designated the current year, 2020, as the "International Year of the Nurse and Midwife." The purpose of said designation was to highlight the magnificent impact of nursing on world health. On many occasions, the WHO continues, nurses are the "first and only point of care in their communities" [27]. In fact, they also emphasize the need for nine million more nurses and midwives in order to meet the healthcare demands of the world community. The International Council of Nurses (ICN) [28] defines the nurse practitioner/advance practice nurse as a: Registered nurse who has acquired the expert knowledge base, complex decision-making skills and clinical competencies for expanded practice, the characteristics of which are shaped by the context and/or country in which $\mathrm{s} / \mathrm{he}$ is credentialed to practice. A master's level degree is recommended for entry level [28].

In order for the deficit of nurses to decrease, it is in the advance practice nurse's role to execute transformative changes based on nursing research, therefore 1) more nurses will work longer, and 2) new nurses are attracted to safe working environments that welcome their voices [29].

Additionally, the advance practice registered nurse (APRN) has the education and experience to create effects in health system policy and state/federal legislation. Once the APRN has completed projects that bring about improvements to the workplace, s/he can take those stories and the evidence to department managers', state and federal legislators' desks to enact innovations to promote healthy working environments for operating room staff for years to come [30]. APRNs' experiences are vast and involve different roles held throughout their meaningful careers, many times unofficially, including responsibilities requiring high levels of leadership, teamwork, research and education, project management and clinical practice.

\section{Limitations}

Due to time constraints, circulating nurses, surgical technicians, and surgical assistants attended the education and in-service training; however, the surgeons, residents, anesthesiologists, CRNAs, and physician assistants were not in attendance. The cardiology and part of the neurology department did not partake in the pre-survey because of the anticipated bulkiness and the thicker electrocautery handle when adding the filter component which encouraged the team discussion of having more than one option of filter devices for a surgical department that performs different types of surgeries requiring a variety of techniques.

Additionally, the project team did not include surgical suction as a possible method to decrease the amount of surgical smoke as part of the survey (in item \#5) which could have provided an accurate assessment of the staff's perception of pre-intervention effective smoke evacuation methods. Three respondents wrote in that response onto the pre-survey and those responses were added to the data analysis. It also would have been important to assess the level of education of each of the participants, to recognize any patterns between that and each person's perception of the dangers of surgical smoke exposure and their willingness to increase the use of filter device.

\section{Recommendations}

It is the project team's recommendation that each hospital create surgical smoke safety policies for the protection of its staff and patients. This is the time to involve APRNs to develop clear guidelines for the protection of the healthcare workforce. Daily, APRNs work with multiple disciplines. They understand the needs of patients, staff, institutions and regulatory entities. APRNs have the education, flexibility and experience to handle the ever-changing landscape of the nursing and surgical nursing world. Surgical smoke protection should be a component of the hiring process of every operating room worker.

Based on the team's observations, it would also be recommended that all members of surgical teams become involved in choosing smoke evacuation devices specific to their specialty, surgical techniques, experience, types of procedures, anatomical location and comfort. Many of the surgeons who participated appreciated the implementation of the filter and were also interested in filter devices that better fit their needs. Those who didn't participate were those who held firmly to their perceptions that the designated filter device would not work for their purposes. In agreement with AORN, it is imperative that surgical teams work with management, clinical engineers, materials managers and infection preventionists to assist in this endeavor.

It is also recommended for future researchers to survey the staff about their medical history, actual symptoms and correlate those with the time spent in an operating room. It would also be beneficial for current and future surgical teams 
to see how that symptomatology relates to staff retention and absenteeism, job satisfaction, patient satisfaction and patient outcomes. Also, surgical teams should have access to or be mandated to wear devices-much like radiology staffto measure and limit exposure to toluene and benzene, for instance.

Lastly, because surgical teams vary in experience, tools, education and leadership styles, and some surgical departments focus on certain procedures more than others, quality improvement projects carried out at multiple operating room sites by nurses of all educational levels interested in improving work environments would provide the necessary data that can be reproducible at a variety of sites. Programs like NIOSH's Total Worker Health can benefit from the findings of those projects to advance the science that has the potential to create the overhaul necessary to protect the operating room workforce from the dangers of surgical smoke exposure. Dr. Paul A. Schulte, in his November 2019 webinar, discussed exposomics, which he defined as the study of the totality of worker exposures. He recognized that work is a primary factor for anyone's health and that a holistic approach is required to prevent illness, advance wellbeing and therefore increase productivity [31].

\section{Conclusion}

It is crucial for teams to discuss ways in which their lives are affected by their work environments. As was observed in this exercise, many of the different roles, including the surgeons, shared similar dissatisfaction with pre-intervention methods of smoke reduction in the operating room. It is critical for healthcare workers to commit to doing their own research. There were many respondents who mentioned symptoms that were decreased once the filter devices were used. The circulating nurses were under the impression that surgeons preferred not to use any electrocautery filter device, when in fact, surgeons seem to prefer a filter device that is more suited to their comfort level, procedure type, field visualization and low noise levels, which allows them to focus on the work they must complete.

Since the implementation of the electrocautery filter device, many of the staff expressed positive views about its use. The use of the filter device has increased, and the OR staff is working together with the other specialties and departments to offer other filter options. In addition to the tangible effects of experiencing less symptoms of nausea, there must be a sense of care that was transmitted to the operating room staff that will probably continue to be expressed as they advance in their quest for a healthier OR environment. The Association of Critical-Care Nurses' (ACCN) [32] policy, in fact, states that healthy environments enable nurses to provide high levels of compassionate care, which is work that fulfills the nurse (ACCN, n.d.). The tenets of that policy: skilled communication, true collaboration, effective decision-making, appropriate staffing, meaningful recognition, and authentic leadership are all standards that were observed throughout the quality improvement project.

\section{References}

1. Saito AC, Margatho AS, Bieniek AA, Stanganelli NC, Ribeiro RP (2019) Signs and symptoms related to inhalation of surgical smoke in the nursing team. Escola Anna Nery 23(3).

2. Okoshi K, Kobayashi K, Kinoshita K, Tomizawa Y, Hasegawa S, et al. (2015) Health risks associated with exposure to surgical smoke for surgeons and operation room personnel. Surgery Today 45(8): 957-965.

3. Association of periOperative Registered Nurses (2019) Colorado second state to enact surgical smoke evacuation law.

4. Lindsey C, Hutchinson M, Mellor G (2015) The nature and hazards of diathermy plumes: A review. AORN Journal 101(4): 428-442.

5. York K, Autry M (2018) Surgical Smoke: Putting the Pieces Together to Become Smoke-Free. AORN Journal 107(6): 692-703.

6. Katoch S, Mysore V (2019) Surgical smoke in dermatology: Its hazard and management. Journal of Cutaneous and Aesthetic Surgery 12(1): 1-7.

7. Tan E, Russell K (2017) Surgical plume and its implications: A review of the risk and barriers to a safe workplace. Journal of Perioperative Nursing 30(4): 3335.

8. Osman JB (2016) Surgical smoke danger: Time for consistent policy and practice. The Dissector 43(4): 1420.

9. Bree K, Barnhill S, Rundell W (2017) The Dangers of electrosurgical smoke to operating room personnel: A review. Workplace Health \& Safety 65(11): 517-526.

10. Joanna $H(2009)$ Introduction to health behavior theory. $2^{\text {nd }}($ Edn.), In: Jones, Bartlett, et al. pp: 1-10.

11. Occupational Safety and Health Administration. Toluene: Occupational exposure limits.

12. Lee T, Soo J, LeBouf RF, Burns D, Schwegler-Berry D, et al. (2018) Surgical smoke control with local exhaust ventilation: Experimental study. Journal of Occupational and Environmental Hygiene 15(4): 341-350. 
13. Schultz L (2014) An analysis of surgical smoke plume components, capture and evacuation. AORN Journal 99(2): 289-298.

14. American Cancer Society (2019) Known and probable human carcinogens.

15. Tseng HS, Liu SP, Uang SN, Li-Ru Yang, Lee LR, et al. (2014) Cancer risk of incremental exposure to polycyclic aromatic hydrocarbons in electrocautery smoke for mastectomy personnel. World Journal of Surgical Oncology 12(1): 1-16.

16. Dobbie MK, Fezza M, Kent M, Lu J, Saraceni ML, et al. (2017) Operation Clean Air: Implementing a Surgical Smoke Evacuation Program. AORN Journal 106(6): 502512.

17. Asdornwised U, Pipatkulchai D, Damnin S, Chinswangwatankul V, Boonsripitayanon M, et al. (2018) Recommended practices for the management of surgical smoke and bio-aerosols for perioperative nurses in Thailand. Journal of Perioperative Nursing 31(1): 33-41.

18. Rioux M, Garland A, Webster D, Reardon E (2013) HPV positive tonsillar cancer in two laser surgeons: case reports. Journal of Otolaryngology-Head \& Neck Surgery 42(1): 54 .

19. Holmes S (2016) Factors affecting surgical plume evacuation compliance. ACORN: The Journal of Perioperative Nursing in Australia 29(4): 39-42.

20. Kochera G, Sesiaa S, Lopez-Hilfikerb F, Schmida R (2019) Surgical smoke: Still an underestimated health hazard in the operating theatre. European Journal of CardioThoracic Surgery 55: 626-631.

21. Liu N, Filipp N, Wood KB (2019) The utility of local smoke evacuation in reducing surgical smoke exposure in spine surgery: a prospective self-controlled study. The Spine Journal.
22. Occupational Safety and Health Administration. Acrylonitrile: Occupational exposure limits.

23. Association of perioperative Registered Nurses (2017) Guideline Summary: Surgical Smoke Safety. AORN Journal 105(5): 498-500.

24. Hirshon JM, Risko N, Calvello EJ, Stewart de Ramirez S, Narayan M, Theodosis C, et al. (2013) Health systems and services: The role of acute care. Bulletin of the World Health Organization 91(5): 386-388.

25. Jayasekara R (2012) Focus groups in nursing research: Methodological perspectives. Nursing Outlook pp: 60.

26. McNicholas A, McCall A, Werner A, Wounderly R, Marinchak E, et al. (2017) Improving patient experience through nursing satisfaction. Journal of Trauma Nursing 24(6): 371-375.

27. World Health Organization (2020) Year of the nurse and the midwife 2020.

28. International Council of Nurses (2020) Definition and characteristics of the role: Nurse practitioner and advance practice roles.

29. Smith NRMC, Karakashian ARB (2018) Empowerment and job satisfaction in nurses. CINAHL Nursing Guide.

30. Chilton L (2015) Nurse practitioners have an essential role in health policy. The Journal for Nurse Practitioners 11(2): A19.

31. Schulte PA, Cauda E, Sahmel J (2019) Where exposure meets sensor technologies: A look at the opportunities and complexities. In NIOSH Total Worker Health Webinar Series.

32. (2020) American Association of Critical-Care Nurses. Healthy Work Environments. 